Instituto Internacional de Investigación y Desarrollo Tecnológico Educativo INDTEC, C.A.

DOI: https://doi.org/10.29394/Scientific.issn.2542-2987.2020.5.15.11.229-246

OAI-PMH: http://www.indteca.com/ojs/index.php/Revista Scientific/oai

Artículo Original / Original Article

\title{
Valores éticos y emociones desde el desarrollo de metodologías activas en la formación docente
}

\author{
Autores: Madelin Rodríguez Rensoli \\ Universidad Nacional de Educación, UNAE \\ madelin.rodriguez@unae.edu.ec \\ Cuenca, Ecuador \\ https://orcid.org/0000-0002-8591-1561 \\ Wilfredo García Felipe \\ Universidad Nacional de Educación, UNAE \\ wilfredo.garcia@unae.edu.ec \\ Cuenca, Ecuador \\ https://orcid.org/0000-0001-6235-6828 \\ Claudia Fuentes Rodríguez \\ Universidad Católica de Cuenca, UCACUE \\ fuentesrodriguezclaudia@yahoo.com \\ Cuenca, Ecuador \\ https://orcid.org/0000-0003-4564-6422
}

\section{Resumen}

El desarrollo de las metodologías activas ha sido objeto de análisis teórico y práctico en esta propuesta, como necesidad de que sean los estudiantes los principales protagonistas del proceso enseñanza aprendizaje, dejando de ser receptores de conocimientos para convertirse en activos productores de nuevos saberes. Se ha constatado que estas, por sí solas, no logran el impacto deseado si no se emprende la relación valores éticos - emociones, siendo recogida esta primera experiencia en el libro: Educación, ética y valores. Otra mirada desde la práctica educativa, publicado en el año 2018. Por ende, el objetivo de esta investigación fue demostrar desde contextos y períodos diferentes los resultados similares que se alcanzaron en el aprendizaje de los docentes desde esta relación. Se utilizó una metodología de observación participante desde los criterios de polaridad de las tablas de diferencial semántico para evaluar estados emocionales desde un diseño de investigación acción participativa. Los resultados análogos alcanzados a partir de evaluaciones organizadas en tres períodos y contextos diferentes, permitieron arribar a un principio pedagógico, que favorece valores éticos, demostrando así, la repercusión y prioridad que tienen las emociones, en el desarrollo de los aprendizajes y el fomento de los valores en los sujetos.

Palabras clave: ética; sentimiento; metodología.

Cómo citar este artículo:

Rodríguez, M., García, W., \& Fuentes, C. (2020). Valores éticos y emociones desde el desarrollo de metodologías activas en la formación docente. Revista Scientific, 5(15), 229-246, e-ISSN: 25422987. Recuperado de: https://doi.org/10.29394/Scientific.issn.2542-2987.2020.5.15.11.229-246

Fecha de Recepción: 28-08-2019
Fecha de Aceptación: 09-12-2019
Fecha de Publicación: 05-02-2020 


\title{
Ethical values and emotions from the development of active methodologies in teacher training
}

\begin{abstract}
The development of active methodologies has been the object of theoretical and practical analysis in this proposal, as the need for students to be the main protagonists of the teaching-learning process, ceasing to be knowledge recipients to become active producers of new knowledge. It has been found that these, by themselves, do not achieve the desired impact if the relationship between ethical values - emotions is not undertaken, this first experience being collected in the book: Education, ethics and values. Another look from the educational practice, published in 2018. Therefore, the objective of this research was to demonstrate from similar contexts and periods the similar results that were achieved in the learning of teachers from this relationship. A participant observation methodology was used from the polarity criteria of the semantic differential tables to evaluate emotional states from a participatory action research design. The analogous results obtained from evaluations organized in three different periods and contexts, allowed us to arrive at a pedagogical principle, which favors ethical values, thus demonstrating the impact and priority that emotions have, in the development of learning and the promotion of the values in the subjects.
\end{abstract}

Keywords: ethic; feeling; methodology.

\section{How to cite this article:}

Rodríguez, M., García, W., \& Fuentes, C. (2020). Ethical values and emotions from the development of active methodologies in teacher training. Revista Scientific, 5(15), 229-246, e-ISSN: 2542-2987. Recovered from: https://doi.org/10.29394/Scientific.issn.2542-2987.2020.5.15.11.229-246

Date Received:

28-08-2019
Date Acceptance:

09-12-2019
Date Publication: 05-02-2020 


\section{Introducción}

La calidad del proceso de enseñanza y aprendizaje constituye una ocupación y preocupación en los diferentes sistemas educativos a nivel internacional. En este sentido, el poco protagonismo de los estudiantes en la producción de nuevos saberes constituye un desafío para el alcance de la excelencia educativa y para responder a una formación adecuada para la vida, tomando en consideración que todo proceso de aprendizaje, desde los primeros años de vida, constituye un eje central en la formación del hombre como ser social e individual.

La llamada crisis de los valores en las sociedades impacta en las instituciones educativas; pues la globalización, no de la solidaridad, sino de hegemonismos - nada beneficiosos para las grandes mayorías - erosiona el proceso de formación de la nueva generación que hoy está en las aulas. Tales presupuestos descansan bajo el criterio del periodista y escritor uruguayo Galeano (2014a), cuando expresaba que: "[...] el mundo al revés premia al revés: desprecia la honestidad, castiga el trabajo, recompensa la falta de escrúpulos y alimenta el canibalismo. Sus maestros calumnian a la naturaleza: la injusticia, dicen, es la ley natural" (pág. 5).

No sería realidad ajena, entonces, que tal situación exigiese un repensar de la Educación en toda su dimensión, uno que sobrepasase el mero hecho de instruir o abordar contenidos de las diferentes asignaturas que en los currículos se plasman, para englobar todo un sinnúmero de plataformas en donde el estudiante sea el protagonista y hacedor de conocimientos. Este repensar de la Educación, entre otras de sus aristas, requerirá analizar, sin duda alguna, cuáles serán las metodologías a seguir, qué métodos son los más apropiados a utilizar y cómo asumir el rol de docente y de estudiantes en el quehacer sistemático y en cada una de las unidades educativas, siempre en pos de facilitar el fomento de una formación ciudadana con pertinencia social.

Dando continuidad al pensamiento de Galeano (2014b): "el mundo al 
revés nos enseña a padecer la realidad en lugar de cambiarla, a olvidar el pasado en lugar de escucharlo y a aceptar el futuro en lugar de imaginarlo" (pág. 8). Y es que, sin lugar a dudas, es la ética y el compartir valores éticos lo que puede direccionar a la Educación en el cumplimiento de lo que puede hacerse llamar su "Misión Social".

Desde este prisma, fue que se inició y desarrolló la investigación, que no solo plasma el resultado de un ambiente que propició, en todo momento un espacio de aprendizaje mutuo y permanente, sino que también quedó revelada la afirmación de que el compartir valores se convierte en un sueño realizable, en la que una premisa fundamental que debe sustentarla lo es, la interrelación que debe lograrse entre el compromiso y el liderazgo colaborativo durante la gestión. Realidad palpable a través de procesos de intervención que favorecieron, desde la observación participante, arribar a un principio básico, desde la constatación personal de los autores en contextos diversos.

La enseñanza y aprendizaje en procesos de formación se constituyó en mediadora para afirmar, una vez más que, en la formación integral del ciudadano, en este caso de docentes, tiene que prevalecer la integración emociones-valores éticos-metodología activa, que permita jerarquizar los sentimientos de los participantes como centro y eje de cualquier actividad.

Es de esta manera como, en la actualidad, se convierte en un hecho que el adecuado manejo de las emociones esté íntimamente relacionado a la motivación en el alumno; influyendo de manera significativa, entonces, en la calidad del conocimiento que se adquiere. Asimismo, se reafirma la relación existente entre el contexto y la experiencia personal de cada individuo, así como sus capacidades de autocrítica, regulación emocional y autoconocimiento en el proceso cognitivo, generando como consecuencia la optimización y revitalización de la enseñanza en cualquier espacio educativo.

En palabras de Dussel (2000):

[...] La ética tiene que ver con la vida y la muerte de la 
humanidad. Si no tenemos un cierto criterio ético, vamos a hacer que la vida siga el camino de un suicidio colectivo [...]. La ética no trata de los actos buenos o malos, sino de las honestas condiciones de posibilidad, de la pretensión de la bondad de un acto: yo creo que este acto es bueno porque pretendo que es verdadero, válido y factible, si usted demuestra lo contrario lo modifico porque soy honesto (pág. 42).

$\mathrm{Y}$, en total acuerdo con lo citado anteriormente, se convierte la Educación en un espacio, en la que se hace realidad el sentir felicidad desde el yo en interrelación con los otros, siendo la actitud positiva de los docentes ante el cambio, lo que permitirá la creación de un escudo moral capaz de enfrentar modelos económicos sociales que pretendan reducir el impacto de la Educación en los procesos de formación.

Conscientes, entonces, de la necesidad de fomentar una actitud ética, ha de asumirse que los valores éticos deben ser compartidos en las instituciones educativas, complementando los objetivos a lograr en cada nivel de enseñanza, conjuntamente con las metodologías activas que se implementen en el proceso de enseñanza y aprendizaje.

En el cómo hacerlo está el gran reto para los colectivos pedagógicos, pues lamentablemente el formalismo y la carencia de toma decisiones por consenso son capaces de convertir el buen propósito en un boomerang para las instituciones educativas carentes del diálogo y la consulta sistemática. Se requiere, por tanto, un liderazgo creíble, el cual se obtiene por la conducta y pautas en forma de ideas éticas.

Para Barylko (2005): y otros diversos autores dedicados al estudio de la ética, no hay creencia o idea sino antecede a ella una cierta lógica. Un fundamento o presupuesto íntimamente vinculado a la cultura, a la sociedad, al contexto y al momento histórico en el que se desarrolla el individuo. Constituyen estas, pautas para su accionar y, consecuentemente, detrás de 
cada conducta se ve reflejado un mundo subjetivo que responde, entre otras cosas, al sentido de la ética y la capacidad de su divulgación (pág. 13).

Es innegable, sin pretender ser absolutos, que cuando existen directivos que promueven la democracia participativa y protagónica en el proceso de dirección educacional con una actuación ética; rebasan la relación de subordinación por la de cooperación y colaboración con los que dirigen; logran consenso en las principales decisiones que se adoptan en la institución educativa $y$, sin lugar a dudas, multiplican su liderazgo, comprometiendo a todos con los resultados.

Todo ello reafirma la necesidad de potenciar la actitud ética, si se pretende que la nueva generación asuma el rol que le corresponde y emerja como generador de cambios. Transformaciones no solamente desde el punto de vista personal, sino también social, mundial, en pos de una cultura de compasión y colaboración con el otro, con la naturaleza, con el medio que nos rodea. Es, en este laborioso andar, que la Educación y quienes forman parte de ella se descubren en una disyuntiva, mediante la cual o bien asume un rol como contribuidor a la formación integral de los ciudadanos, o se convierte en cómplice de un final donde la equidad y la falta de justicia social se apoderan como aliados de la comunidad.

Sin embargo, cuando se habla de valores éticos compartidos, y de enseñanza y aprendizaje, es importante no olvidar las emociones. Afirma Gendron (2009): que el docente enfrenta en su quehacer profesional situaciones de tensión que tienen una gran influencia en sus estados emocionales, y no solo el de él, sino en sus estudiantes, y el propio proceso de enseñanza y aprendizaje, se puede citar, exceso de trabajo, problemas personales, falta de tiempo, entre otras (pág. 7). Por ende, asumir la profesión de docente, implica comprender que no es solo un acto cognitivo, sino de afectos, emociones, sentimientos, que influyen directamente en la calidad de la educación. Los estados emocionales, los valores éticos compartidos y la 
educación, son parte de nuestras propias vidas.

Además, Terán, Perdomo y Castillo (2017), sostienen que:

[...] La educación busca la evolución, transformación e inmortalidad del ser humano; la enseñanza forma, prepara, desarrolla, perfecciona al hombre en su vida, en su convivencia social, lo ayuda a encontrarse, así mismo, a proyectarse ante los demás; por ello, la educación le da un orden ético, moral y social al ser, todo ello aunado a su pensamiento (pág. 31-32).

Por consiguiente, se requiere que la Educación sea direccionada no solo al aprendizaje de diversos contenidos específicos, ampliados por la actividad autónoma del estudiante, sino que también trascienda mucho más allá de la simplicidad de una teoría, alcanzando el desarrollo personal, de sentimientos de felicidad, solidaridad, que contribuya a disminuir sensiblemente la falta de equidad e injusticia social.

En este sentido, el objetivo de este trabajo está dirigido a: demostrar la integración que existe entre las emociones-valores éticos-metodología activa, para el logro de una educación de calidad, desde un enfoque transdisciplinar en los procesos de gestión de las Instituciones educativas en vínculo con el territorio.

\section{Metodología}

Teniendo en cuenta los enfoques y tipologías de diseños que plantea Hernández, Fernández y Baptista (2014): se asume en este proyecto un enfoque cualitativo y un tipo de diseño de investigación acción participativa. Es importante destacar que además de propiciar la transformación de la realidad de ese contexto, se contribuyó a que los involucrados tomaran conciencia de su rol en este proceso (págs. 470-496). Bajo esta, se utilizaron métodos típicos del nivel teórico, empírico y matemático-estadísticos que posibilitaron cumplir con los objetivos declarados. En este caso, la observación participante se desarrolló con el auxilio de tablas de diferencial semántico de actitudes, 
valores y estados emocionales.

Es importante destacar que la investigación acción participativa que sustentó dichos resultados, requirió de consultas sistemáticas para la toma paulatina de decisiones con eficiencia y eficacia, existiendo la seguridad por los autores de esta ponencia, de que a pesar de que la lógica fue construida desde la concepción de una práctica educativa, su introducción en un grupo de instituciones la enriqueció.

Para la realización de este estudio, se definieron períodos relacionados con los momentos en que se evaluaron los estados emocionales, en procesos de formación docente:

- Un primer período centrado en acciones de formación continua a un total de 100 docentes, durante la etapa de junio-diciembre del año 2016, en Lago Agrio, provincia de Sucumbíos.

- Un segundo período centrado en acciones de formación continua a un total de 58 docentes de las provincias Azuay y Cañar, durante la etapa de mayo-julio del año 2017, en los cantones, Cuenca y Azogues.

- Un tercer período, desde las experiencias implementadas en el año 2019, como parte de los trabajos de culminación de estudios universitarios en la carrera de Educación Básica por Pérez y Sacta (2019a): durante un proceso de formación continua a 20 docentes en la etapa mayo-agosto, en el Cantón Cuenca, Provincia Azuay (pág. 66).

Se organizaron varias fases, que favorecieron la identificación de resultados en cada contexto, las cuales se concretaron en:

Fase 1. Determinación de la población a observar en cada uno de los contextos y períodos en las que se desarrollaron los programas de formación continua.

Fase 2. Aplicación de las Tablas de Diferencial Semántico (TDS), en encuentros iniciales, intermedios y finales, que permitieran identificar los 
cambios que se producían en cada intervención.

Fase 3. Realizar el análisis comparativo de los resultados obtenidos en cada período de los declarados.

Fase 4. Arribar a conclusiones.

Desde los resultados análogos obtenidos en los tres períodos, la dinámica de las relaciones interpersonales que se producían durante el desarrollo de las actividades en cada uno de ellos, el desarrollo de acompañamientos en las escuelas desde la implementación de la Lesson Study, en contextos diferentes, favorecieron la determinación de los resultados que se presentan.

En este caso se asumen los acompañamientos desde la Lesson Study, por las relaciones interpersonales, de colaboración y cooperación que se logran en un grupo de docentes que enfrentan una profesión y problemas comunes, desde la confianza, en asumir posiciones críticas y conscientes, teniendo en cuenta procedimientos y vías para contribuir a la solución de los problemas desde una reflexión profunda de las consecuencias de los problemas de su práctica profesional.

\section{Resultados}

Durante el primer período se observó un tránsito a la polaridad positiva, como refleja el gráfico 1, en la que se manifestaba, desde la implementación de metodologías activas, y un liderazgo que favorecía la participación entre iguales, un cambio significativo en los modelos de pensamiento, desde el compromiso consigo mismo y de orientar su actividad desde los sentimientos. Un elemento importante a considerar, lo constituyó, la disminución del aislamiento entre los docentes, la sensibilización en la necesidad de cooperar y colaborar en busca de las respuestas a las problemáticas planteadas. 
Gráfico 1. Evaluación de actitudes (forma de trabajo y disposición), estados emocionales y valores en 100 docentes que transitaron por el Programa de Educación Continua de la Universidad Nacional de Educación (UNAE), Amazonía.

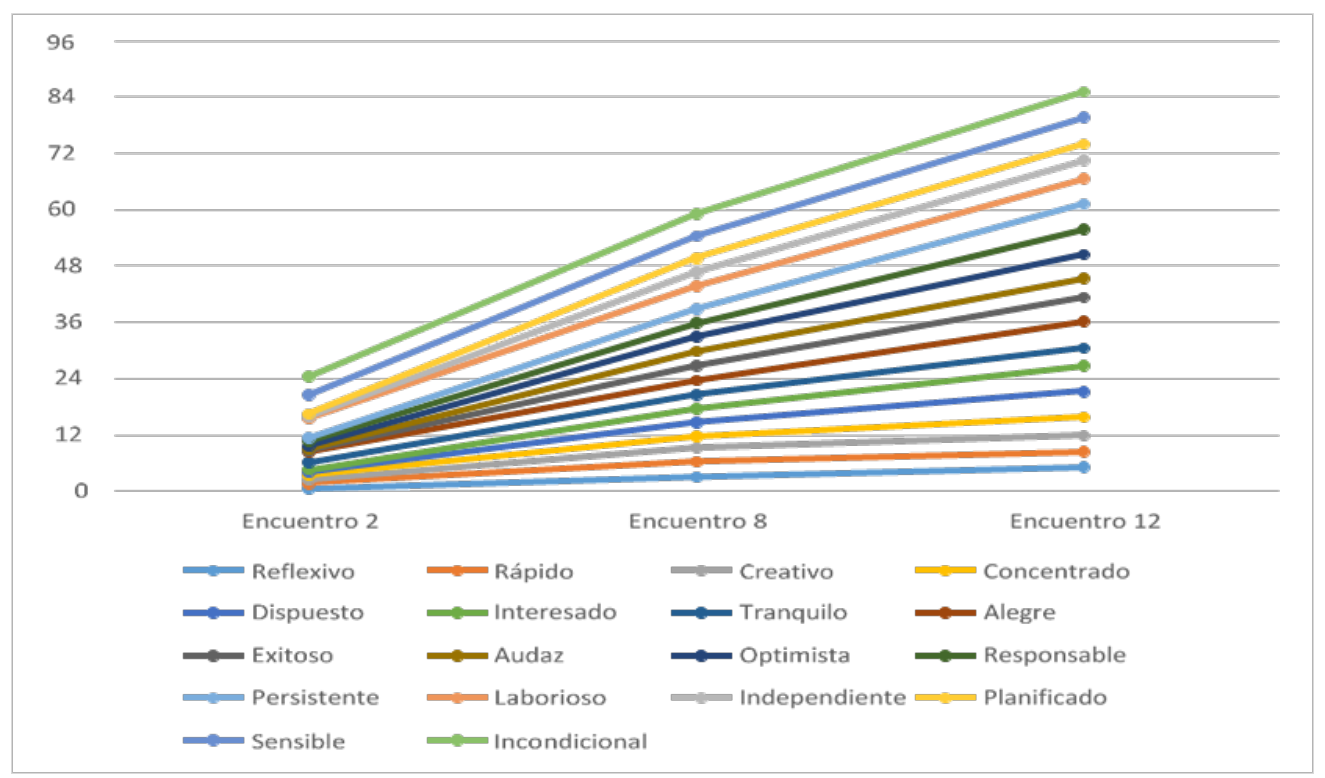

Fuente: Los Autores (2019).

En el segundo período, a pesar de constituir un contexto diferente, se fueron observando avances medianamente positivos $( \pm)$, como refleja el gráfico 2. $\mathrm{Y}$ aunque mostraron incrementos discretos de sus niveles emocionales, su contribución a la solución de problemas si demostraron lo contrario, a medida que se adentraban en los procesos de análisis y sentían, que su aporte, constituían elementos importantes en la reflexión desde las metodologías activas aplicadas. En el gráfico 1 y gráfico 2, se puede apreciar, que no solo se evalúan estados emocionales, sino que estos se asocian a formas de trabajo, disposición y valores que favorecían desde su complementación, a lograr los resultados esperados. 
Gráfico 2. Evaluación de actitudes (forma de trabajo y disposición), estados emocionales y valores en 58 docentes que transitaron por el Programa de Educación Continua de Cuenca, Azogues.

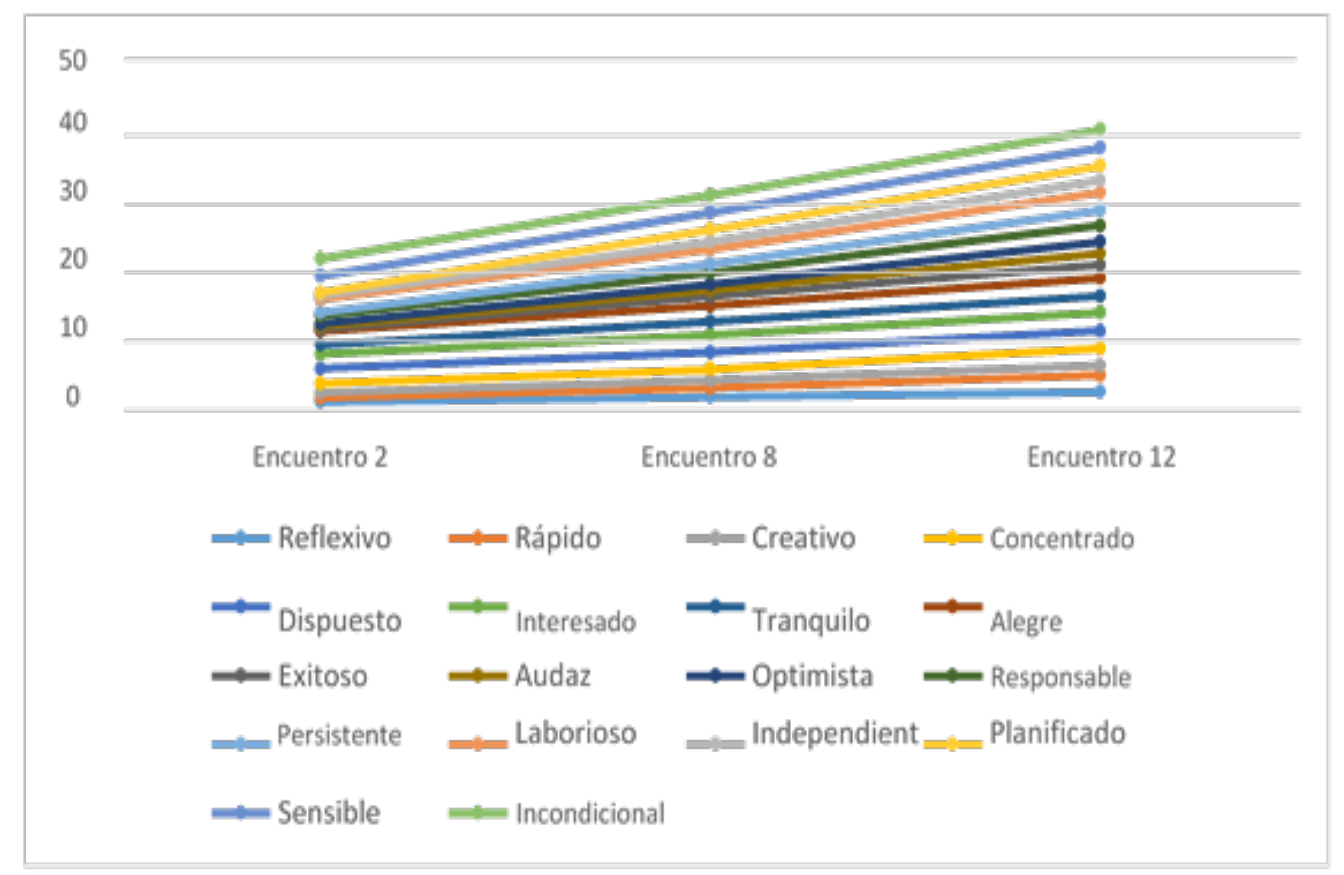

Fuente: Los Autores (2019).

En el caso del tercer período, se centraba en un proceso de formación, de estudiantes que se encontraban desarrollando sus trabajos de culminación de estudios universitarios en la carrera de Educación Básica. Como expresan Pérez y Sacta (2019b): solo se evaluó el criterio de estados emocionales y manifestaron expresiones de satisfacción, risas y vivacidad (págs. 65-66); como refleja el gráfico 3.

De manera que, al enfrentarse a la realidad de los resultados obtenidos, la dinámica de las relaciones interpersonales que se producían durante el desarrollo de las actividades en los diferentes contextos en los que se investigó, se sistematizó la experiencia obtenida, se estaba en condiciones de arribar a consideración de los autores, a un principio pedagógico. 
Gráfico 3. Polarización de estados emocionales durante los 4 encuentros presenciales del programa de formación en Cuenca.

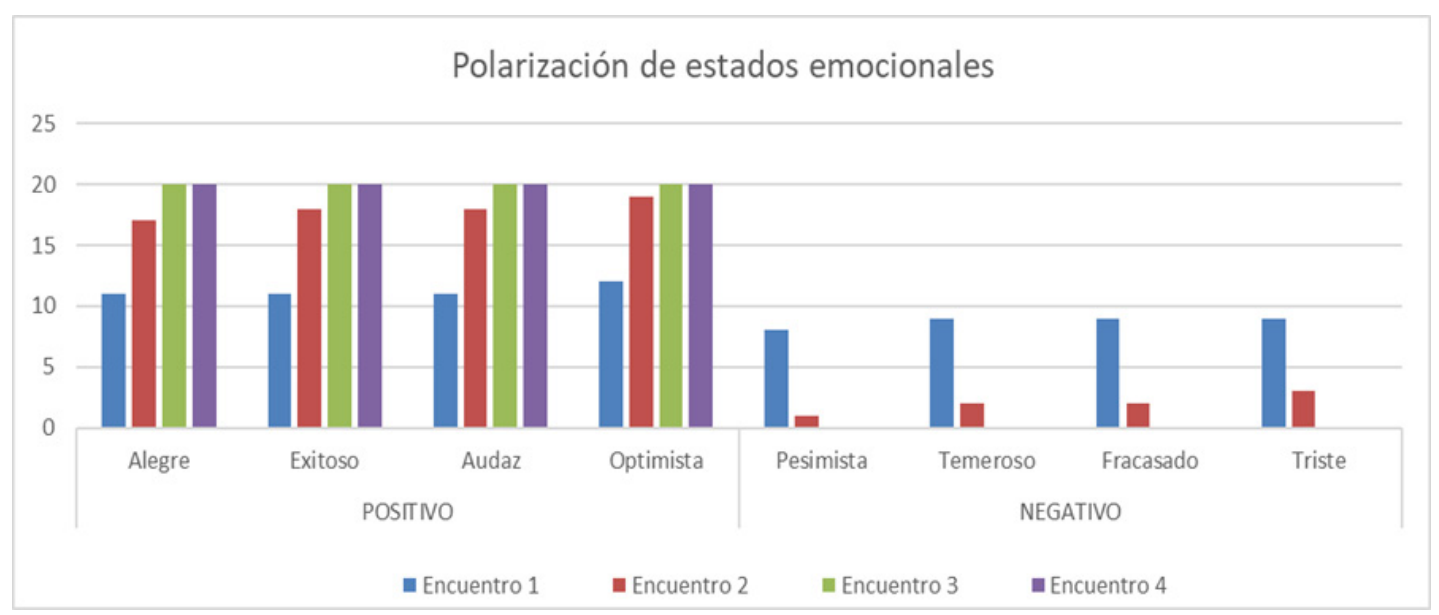

Fuente: Pérez y Sacta (2019).

La fundamentación que derivó en principio, fue la enseñanza y aprendizaje, en procesos de formación, adquiere un impacto positivo, alcanzando un clima afectivo de placer, satisfacción, felicidad antes las tareas a desarrollar y audacia, cuando la comunidad educativa potencia y jerarquiza como primera prioridad las relaciones que se dan entre emociones-valores éticos-metodologías activas, y se transversaliza desde un enfoque transdisciplinar en los procesos de gestión de las Instituciones educativas en vínculo con las entidades y organizaciones que se encuentran en su territorio. De ahí, que estos fundamentos posibilitaron el alcance del principio, bajo la denominación: Principio de integración emociones-valores éticos compartidosmetodologías activas desde un enfoque transdisciplinar en los procesos de gestión de las Instituciones educativas en vínculo con las entidades y organizaciones que se encuentran en su territorio. 
Las acciones que sistematizadas generaron el principio pedagógico declarado fueron:

- Diagnosticar las fortalezas y debilidades de la comunidad educativa, no solo en los aspectos cognitivos, sino afectivos-emocionales, en la que debe predominar esta última.

- Propiciar la dinámica del proceso pedagógico de la Institución educativa a partir de las potencialidades identificadas en los procesos de gestión de la dirección y de la enseñanza y aprendizaje, teniendo en cuenta los enfoques pedagógicos que se asumen.

- Estimular la manifestación crítica reflexiva ante el conocimiento de problemas globales en la esfera de la educación, y su vínculo con los que afronta la Institución educativa, partiendo del ejemplo y de un clima afectivo que potencia las relaciones interpersonales.

- Provocar la reflexión de las consecuencias y secuelas de los problemas que enfrenta la Institución educativa, tanto en las esferas de gestióncognitiva-afectiva vinculándolas con la necesidad de potenciar los valores éticos compartidos.

- Estimular el emprender un proceso de gestión desde situaciones problémicas, desde la relación emociones-valores éticos-metodologías activas.

En consecuencia, este principio y sus acciones, se constituyeron en fuentes generadoras de transformaciones de actitudes ante un clima emocional positivo que favoreció el cumplimiento de los objetivos propuestas.

\section{Conclusiones}

El logro de una formación integral en la institución educativa, reflejada en una interacción entre la instrucción y la educación, será posible si se 
complementa el efecto de los objetivos, con el fomento de la ética en el modo

de actuación de la comunidad educativa. La potenciación de las metodologías activas en el proceso de enseñanza aprendizaje, tendrá el efecto esperado en la producción de los nuevos saberes con pertinencia social, si se cultivan en el proceso de formación valores éticos, teniendo en cuenta el desarrollo de un sistema de influencias educativas coherentes, donde la ética rija el modo de actuación de cada miembro de la comunidad educativa, lo que constituye una prioridad de primer orden para la formación de un nuevo ciudadano.

Por ello, la implementación de un proceso de democratización en la dirección, comenzando a transitar de una relación de subordinación en la institución educativa, a una relación de cooperación y colaboración que favorezca la participación y reflexión desde la diversidad, constituye una prioridad en el proceso de enseñanza-aprendizaje. Priorizar un clima emocional-afectivo en el desarrollo de cualquier actividad que se planifique, implica las relaciones interpersonales y el desarrollo de valores éticos compartidos.

En este sentido, se demuestra una vez más, la prioridad que se le debe dar a los afectos, sentimientos, emociones, para lograr la transformación positiva del sujeto en la sociedad, que implica no solo asumir un comportamiento ético desde el desarrollo de valores, sino, comprender la sociedad en su conjunto desde un enfoque holístico de integración para la toma de decisiones, que implique no solo el yo, sino él nos-otros. Reconocer la historia de cada individuo, la búsqueda de información que favorezca la comprensión de las actitudes del otro, aprender a ponerse en la posición del otro, constituyen elementos de gran significación para el docente, en su dirección de la enseñanza y aprendizaje. 


\section{Referencias}

Barylko, J. (2005). La Filosofía. Una invitación a pensar. Argentina: Grafica Mps Srl.

Dussel, E. (2000). El reto actual de la ética: Detener el proceso destructivo de la vida. El nuevo proyecto histórico. La Habana, Cuaba: Editorial Ciencias Sociales.

Galeano, E. (2014a,b). Patas arriba. La escuela del mundo al revés. México: Siglo XXI Editores.

Gendron, B. (2009). Leadership et compétences émotionnelles. Dans l'accompagnement au changement. Canada, United States: Presses de l'Université du Québec.

Hernández, R., Fernández, C., \& Baptista, P. (2014). Metodología de la Investigación. México, D.F.: McGraw-Hill / Interamericana Editores, S.A. de C.V.

Pérez, J., \& Sacta, O. (2019a,b). Fortalecimiento del desempeño profesional docente en el proceso de enseñanza - aprendizaje de la Lengua y Literatura. Trabajo de titulación. Azogues, Ecuador: Universidad Nacional de Educación. Recuperado de:

http://repositorio.unae.edu.ec/handle/56000/1035

Terán, C., Perdomo, E., \& Castillo, R. (2017). Un Recorrido por el Pensamiento Educativo hacia la Transformación de la Educación en el Siglo XXI. Revista Scientific, 2(5), 29-48, e-ISSN: 2542-2987. Recuperado de:

https://doi.org/10.29394/scientific.issn.2542-2987.2017.2.5.2.29-48 


\section{Madelin Rodríguez Rensoli \\ e-mail: madelin.rodriguez@unae.edu.ec}

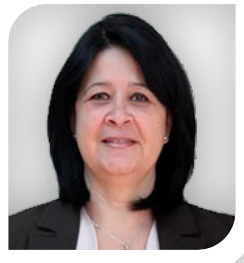

Nacida en la Ciudad de la Habana, Cuba, el 24 de mayo del año 1965. Docente Investigadora Titular Principal 1 de la Universidad Nacional de Educación (UNAE), Ecuador; Doctora en Ciencias Pedagógicas; Máster en Investigación Educativa; Licenciada en Educación, Especialidad Biología; Acreditada como Investigadora Agregada 1 por la SENESCYT en Ecuador; He participado como directora e investigadora en proyectos de investigaciones; Directora de proyectos de investigación, tesis de maestría y doctorado; He publicado en revistas indexadas y en Congresos; Miembro del Consejo Editorial de la Revista Panorama Médico (e-ISSN: 1390-7101); Revista del Instituto Pedagógico Latinoamericano y Caribeño - IPLAC (e-ISSN: 1993-6850); y Gaceta Médica Espirituana (e-ISSN: 1608-8921); Miembro del Equipo de Árbitros de la Revista Scientific (e-ISSN: 2542-2987); He participado como evaluadora externa en procesos de acreditación en Cuba y Ecuador. 


\section{Wilfredo García Felipe}

e-mail: wilfredo.garcia@unae.edu.ec

Nacido en la Ciudad de la Habana, Cuba, el 20 de marzo

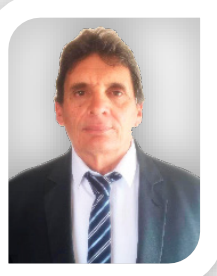
del año 1953. Docente Investigador Titular Principal 1; Doctor en Ciencias Pedagógicas; Máster en Educación Avanzada; Licenciado en Ciencias Sociales; Acreditado como investigador por la SENESCYT en Ecuador; Miembro de Proyectos de Investigación; Director de tesis de Maestrías y Doctorado; He cumplido responsabilidades como docenteinvestigador y directivas en diferentes niveles de educación; He publicado artículos derivados de proyectos de investigación en revistas indexadas; así como capítulos de libros; He participado en investigaciones y colaborado en proyectos sobre gestión educacional, en Congresos Internacionales; Director de tesis de doctorado y de maestrías con temas relacionados a la Didáctica y Pedagogía. 


\section{Claudia Fuentes Rodríguez}

\section{e-mail: fuentesrodriguezclaudia@gmail.com}

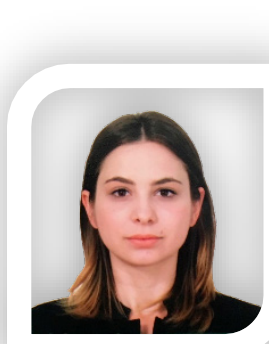

Nacida en Holguín, Cuba, el 13 de agosto del año 1993.

$\mathrm{He}$ sido ponente en eventos con resultados de investigaciones sobre salud y el bienestar psicológico de la población; así como en adultos mayores, relacionadas con el síndrome de Burnout en sujetos dedicados al cuidado del adulto mayor $y$ manifestaciones psicopatológicas de la memoria; Coautora de los libros sobre terapia de pareja y estado mental del adulto mayor; Creadora y guionista del Programa Radial de Salud Mental "Sanamente", en vínculo con la Carrera de Comunicación Social de la Universidad Católica de Cuenca (UCACUE).

El contenido de este manuscrito se difunde bajo una Licencia de Creative Commons ReconocimientoNoComercial-Compartirlgual 4.0 Internacional 